\title{
The Deformation of Cylindrical Shells Subjected to Radial Loads Using Mixed Formulation and Analytic Solutions
}

\author{
Luisa R. Madureira ${ }^{1, *}$, Elza M. M. Fonseca ${ }^{2}$, Francisco Q. Melo ${ }^{3}$ \\ ${ }^{1}$ University of Porto, Faculty of Engineering, Department of Mechanical Engineering, Portugal \\ ${ }^{2}$ Polytechnic Institute of Bragança, Department of Applied Mechanics, Portugal \\ ${ }^{3}$ University of Aveiro, Department of Mechanical Engineering, Portugal \\ *Corresponding Author: luisa.madureira@fe.up.pt
}

Copyright $@ 2013$ Horizon Research Publishing All rights reserved.

\begin{abstract}
The objective of this work is to contribute with a simple and reliable numerical tool for the stress analysis of cylindrical vessels subjected to generalized forces using a mixed formulation. Variational techniques coupled with functional analysis are used to obtain an optimized solution for the shell displacement and further stress field evaluation using a combination of unknown analytic functions with Fourier expansions. A large cylindrical shell subjected to pinching loads is considered. These elements are intended to provide accurate modelling of the initially circular pipes response. Because of this behaviour, the bend's cross-section abandons its original roundness, turning into an oval or noncircular configuration. In addition, the initially plane cross-section, tends to deform out of its own plane. These two deformation patterns are termed ovalization and warping, respectively. In this work the results for the radial displacement and section ovalization are analysed where the solution has six terms for an acceptable accuracy. The transverse displacement presents important dependence on the shell thickness $v s$ radius, where in the case of thin shells the ovalization is restricted to a local area and this is the case analysed. The proposed method leads to accurate results with low complex input data. The conclusions of this work are that the definition of the load system and boundary conditions are easily processed as the method has pre-defined possibilities for each load case or edge boundary conditions. An analytic solution is obtained and a low number of terms in the Fourier series show good accuracy as can be seen by a comparison with finite element methods.
\end{abstract}

Keywords Piping Engineering; Fourier Series; System Of Differential Equations; Boundary Conditions

\section{Introduction}

The solution here proposed deals with the combination of unknown analytic functions with Fourier expansions, where the former depend on the axial shell coordinate and the trigonometric terms are dependent upon the cylinder circumferential polar angle [1]. With this formulation and the evaluation of the total energy a system of ordinary differential equations is obtained and solved where the solution is analytic after evaluation of eigenvalues and eigenvectors. The boundary conditions are then used to achieve the final solution.

The cylindrical shell deformations include axial, circumferential and shear membrane terms $\varepsilon_{x x}, \varepsilon_{\theta \theta}$, and $\gamma_{x \theta}$. In this formulation the shell is assumed to be circumferentially inextensible, so $\varepsilon_{\theta \theta}=0$. Due to the shell elasticity the definition of the displacements in cylindrical thin pipes leads to a deformation field originating internal forces, which for the geometry considered, include the membrane axial force $N_{x x}$, the circumferential force $N_{\theta \theta}$ and the shear force $N_{x \theta}$. and also the bending moments $N_{x x}, N_{\theta \theta}$ and the twist moment $N_{x \theta}$.[1].They are related to the deformations by the elasticity matrix as shown by Eq.(1):

$$
\left\{\begin{array}{l}
N_{x x} \\
N_{x \theta} \\
M_{\theta \theta} \\
M_{x x} \\
M_{x \theta}
\end{array}\right\}=\left[\begin{array}{ccccc}
A & 0 & 0 & 0 & 0 \\
0 & S & 0 & 0 & 0 \\
0 & 0 & D & 0 & 0 \\
0 & 0 & 0 & D & 0 \\
0 & 0 & 0 & 0 & D \frac{1-v}{2}
\end{array}\right]\left\{\begin{array}{l}
\varepsilon_{x x} \\
\gamma_{x \theta} \\
k_{\theta \theta} \\
k_{x x} \\
k_{x \theta}
\end{array}\right\}
$$

The constants $A, S$, and $D$ in Eq.(1) are given by:

$$
A=\frac{E h}{1-v^{2}} \quad S=\frac{E h}{2(1+v)} \quad D=\frac{E h^{3}}{12\left(1-v^{2}\right)}
$$

where $E$ and $v$ are, respectively, the material Young's modulus and Poisson's ratio while $h$ is the pipe thickness (assumed uniform).

The terms presented in Eq.(1) together with internal forces complete the solution unknowns. The displacement field consists of the axial, the circumferential and the transverse shell displacements $u, v$ and $w$ respectively. These displacements result from the superposition of the so-called beam type with the ones assigned to the transverse section distortion. The first group results from the bending of a pipe, 
modelled under current assumptions of a geometrically unchanged section, only undergoing rigid body displacement or rotations. However they are able to generate axial deformations along the pipe axis. The second group of deformations is the most important when radial concentrate or finite area distributed loads are considered. This last type of displacements considers that the transverse section ovalizes and warps. For the same resulting load, the magnitude of the radial displacements compared with the beam type one is much higher; being possible to neglect these last ones if compared with the local shell deformation as mentioned. The shell displacements from radial loads are approached by trigonometric expansions $[2,3,4]$ and are defined in Eq.(3):

$$
\begin{array}{r}
u(x, \theta)=\sum_{i=2}^{n_{\theta}} b_{i}(x) \cos (i \theta) \\
v(x, \theta)=\sum_{i=2}^{n_{\theta}}-\frac{a_{i}(x)}{i} \sin (i \theta) \\
w(x, \theta)=\sum_{i=2}^{n_{\theta}} a_{i}(x) \cos (i \theta)
\end{array}
$$

The functions $a_{i}(x)$ and $b_{i}(x)$ are the amplitudes for the ovalization and warping displacements [4]. All expansions start with index $n=2$ once the order one term refers to the undeformed beam type displacement $[1,2]$. These terms are included in the Fourier $\theta$-expansions, but they are only $x$-dependent functions. $n_{\theta}$ is equal to 6 in the studied example.

The deformation energy $U$ is a functional $U\left(u, v, w, N_{x x}\right.$ $\left.N_{x \theta}, M_{\theta \theta} M_{\mathrm{xx}}, M_{\mathrm{x} \theta}\right)$, for which the minimum is obtained performing a weak formulation with Fourier series amplitude parameter variations. In this problem the terms used for evaluation of the total energy are presented in Eq.(4) as suggested by [4].

$$
\begin{gathered}
U=\frac{1}{2} \iint A \varepsilon_{x x}^{2}+S \gamma_{x \theta}^{2}+D k_{\theta \theta}^{2}+ \\
+\frac{D(1-v)}{2} k_{x \theta}^{2}+D k_{x x}^{2} d \Omega \\
=\frac{1}{2} \iint \sum_{i=2}^{7}\left[A\left(b_{i}^{\prime}(x) \cos i \theta\right)^{2}+S\left(-\frac{i}{r} b_{i}(x)-\frac{a_{i}^{\prime}(x)}{i}\right)^{2}(\sin i \theta)^{2}\right. \\
+\frac{1}{r^{4}} D\left(-i^{2}+1\right)^{2}\left(a_{i}(x) \cos i \theta\right)^{2}+ \\
\left.+\frac{D(1-v)}{2} \frac{\left(-2 i+\frac{1}{i}\right)^{2}}{r^{2}}\left(a_{i}^{\prime}(x) \sin i \theta\right)^{2}+D\left(a_{i}^{\prime \prime}(x)\right)^{2} \cos ^{2} i \theta\right] d \Omega
\end{gathered}
$$

where $d \Omega=r d \theta d x$ and

(the shell axial curvature)

$$
k_{x x}=a^{\prime \prime}(x)_{i} \cos i \theta
$$

(the shell surface twist)

$$
k_{x \theta}=\frac{1}{r}\left(-2 i+\frac{1}{i}\right) a_{i}^{\prime}(x) \sin i \theta
$$

(the circumferential curvature)

$$
k_{\theta \theta}=\frac{1}{r^{2}}\left(\left(-i^{2}+1\right) a_{i}(x) \cos i \theta\right)
$$

(the axial membrane strain)

$$
\varepsilon_{x x}=b_{i}^{\prime}(x) \cos i \theta
$$

(the membrane shear strain)

$$
\gamma_{x \theta}=\left(-\frac{i}{r} b_{i}(x)-\frac{a_{i}^{\prime}(x)}{i}\right) \sin i \theta
$$

Corresponding to a minimum of the functional $U$ [5], separate variations for each of the unknowns $a_{i}(x), b_{i}(x)$ give the following equations:

$$
\begin{aligned}
& \left(\frac{\partial U}{\partial b_{i}(x)} \delta b_{i}(x)+\frac{\partial U}{\partial b_{i}^{\prime}(x)} \delta b_{i}^{\prime}(x)\right)_{i \geq 2}=0 \\
& \left(\frac{\partial U}{\partial a_{i}(x)} \delta a_{i}(x)+\frac{\partial U}{\partial a_{i}^{\prime}(x)} \delta a_{i}^{\prime}(x)\right)_{i \geq 2}=0
\end{aligned}
$$

The solution is now written with totally assumed displacements and its derivatives. Following this procedure, a system of differential equations is obtained. The differential equations for the straight pipe, where the deformation is caused by a force $F$ applied on the surface of the pipe are described in Eq.(8) and solved by evaluation of eigenvalues and eigenvectors [6]:

$$
\begin{gathered}
\pi r D a_{i}^{i v}(x)=-\frac{\left(i^{2}-1\right)^{2} \pi D}{r^{3}} a_{i}(x)+\pi S b_{i}^{\prime}(x)+ \\
\left(\frac{\pi r S}{i^{2}}+\left(-2 i+\frac{1}{i}\right)^{2} \frac{\pi D(1-v)}{2 r}\right) a_{i}^{\prime \prime}(x)+F \\
\pi r A b_{i}^{\prime \prime}(x)=\left(\frac{i^{2} \pi S}{r}\right) b_{i}(x)+\pi S a_{i}^{\prime}(x)
\end{gathered}
$$

\section{Example}

A steel cylinder with rigid end diaphragms is analysed. The cylinder is subjected to opposite loads (pinching mode) applied in the radial direction as shown in Fig.1. This is a benchmark case study frequently analysed by several authors $[7,8]$. The example of a pinched cylinder with the present methodology is compared with the work proposed by [7,9]. Simultaneously, numerical simulation using ANSYS ${ }^{\circledR}$ finite element program will be presented.

In this example lengths are in inches and load in pounds as 
reported in [7,9]. Figure 1 shows the case study and all considered parameters.

The material properties and the geometric parameters are the following (units are according to the references for comparison):

$$
\begin{gathered}
E=3 \times 10^{6} \text { psi } v=0.3 \quad \mathrm{r}=300 \text { inch } \\
\mathrm{L}=600 \text { inch } \quad \mathrm{h}=3 \text { inch }
\end{gathered}
$$

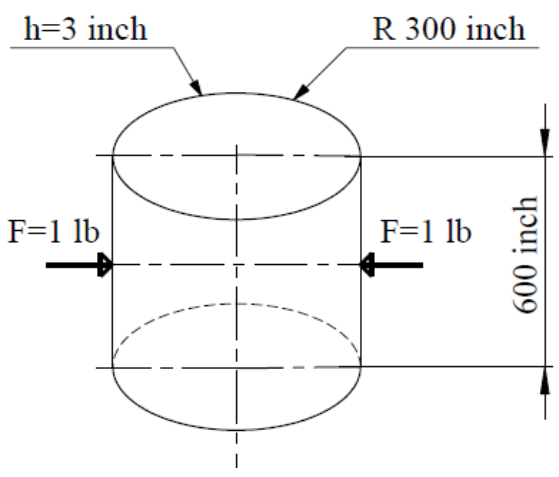

Figure 1. Geometry of a two-radial loads pinched cylinder

\subsection{The Variational Method Application}

Equation 9 represents the load $F$ submitted for this type of analysis and introduced in the differential system given by Eq.(8). A Fourier sin expansion is considered to simulate the point load. It contains odd terms only and for an estimated quadratic error [6] of $6 \%, 59$ terms are used.

$$
\mathrm{F}(\mathrm{x})=\sum_{n=1}^{59} \frac{2 F}{n \pi}\left(-\cos \frac{286 n \pi}{600}+\cos \frac{314 n \pi}{600}\right) \sin \frac{n \pi x}{600}
$$

Figure 2 shows the load considered on the surface as a Fourier expansion.
Superposing the six solutions $a_{2}(x), \ldots, a_{7}(x)$ (Fig.3) obtained from solving Eq.(8) effective solution for the deformation is given. The vertical displacement along the length of the cylinder obtained with this mixed formulation is shown in Fig.4.

The next table (Table 1) shows that these results obtained with the mixed formulation match the ones obtained with finite element methods by $[7,10]$.

Table 1. Vertical displacement (inch)

\begin{tabular}{|c|c|c|}
\hline Fonseca et al [7] & $\begin{array}{c}\text { Dvorkin and Bathe } \\
{[10]}\end{array}$ & Mixed formulation \\
\hline$-1.836 \mathrm{E}-5$ & $-1.752 \mathrm{E}-5$ & $-1.874 \mathrm{E}-5$ \\
\hline
\end{tabular}

\subsection{The finite Element Method Application}

The pinched cylinder problem is useful to assess the accuracy of a structural shell model under bending and membrane states. This study is a benchmark test in the research of the performance of curved beam or shell elements [10]. The finite element method was used for this example. Results are compared with the previous solution obtained with the functional analysis. Displacements obtained using ANSYS $^{\circledR}$ program, are shown in the next figures. A finite Shell93 element was used, with 8 nodes and 6 degrees of freedom per each node: translations in the nodal $x, \mathrm{y}$, and $z$ directions and rotations about the nodal $x, y$, and $z$-axes. Shell93 is particularly well suited to model curved shells. The deformation shapes are quadratic in both in-plane directions. Due the geometry and load symmetry a one-eighth of the cylinder is modelled and all complete cylinder, being the finite element mesh used in Fig.5. For the one-eighth symmetry model, one-fourth of the load is applied.

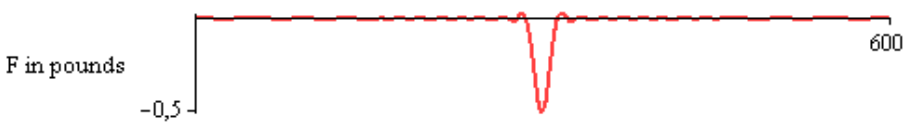

Figure 2. Applied load

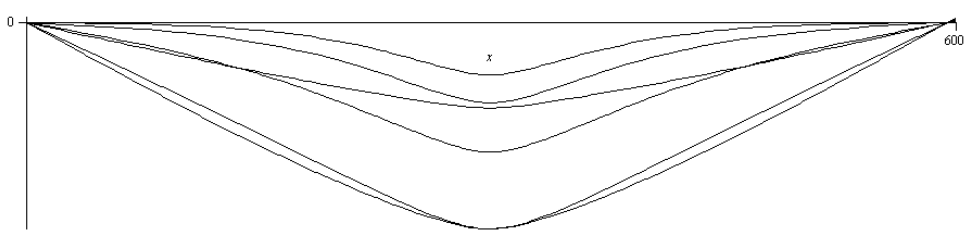

Figure 3. Separate solutions corresponding to each ovalization mode $a_{2}(x), a_{3}(x), \ldots, a_{7}(x)$

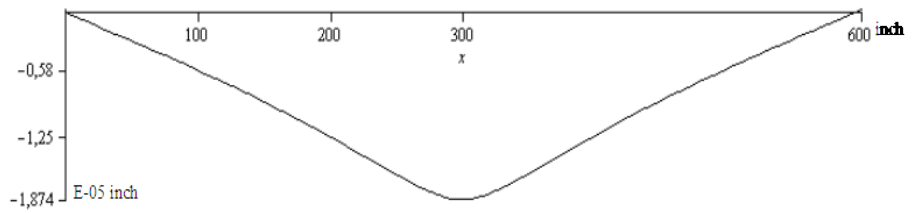

Figure 4. $a_{2}(x)+a_{3}(x)+\ldots+a_{7}(x)$ for pipe deformation 


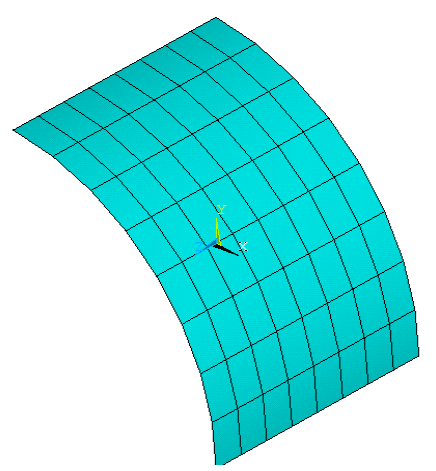

(a)

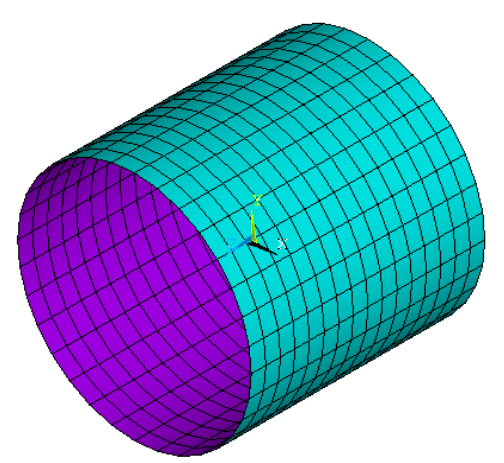

(b)

Figure 5. Finite element mesh used for an eighth (a) and total cylinder length (b)

Figure 6 shows the postprocessor obtained for the vertical displacement results using ANSYS ${ }^{\circledR}$ program.

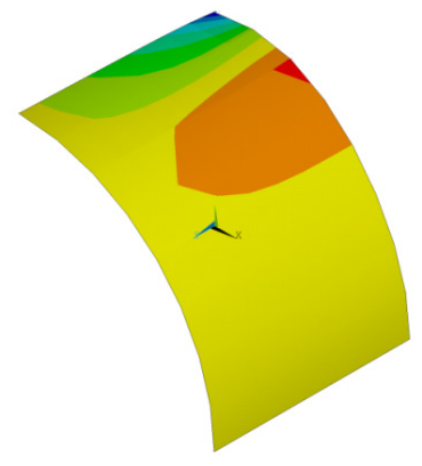

(a)

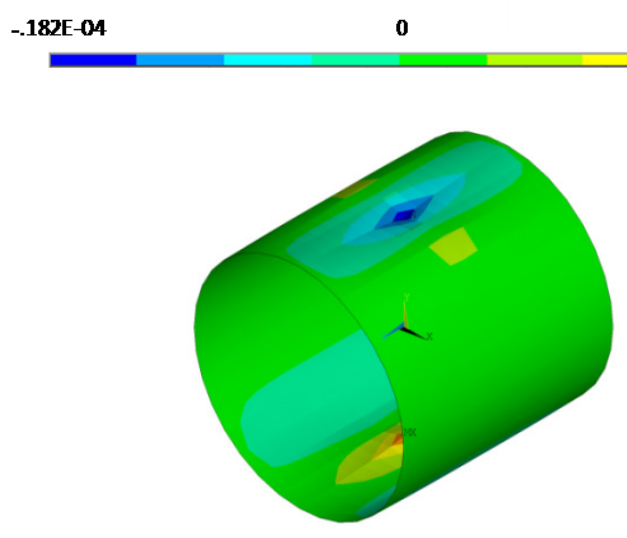

(b)

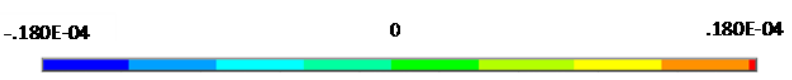

Figure 6. Vertical displacement: a) one eighth; b) total cylinder length
The reference displacement, as reported by $[10,11]$ and the exact deflection at the load point is equal to $0.18248 \mathrm{E}-4 \mathrm{inch}$, using the relation: $\boldsymbol{w}_{\text {exat }}=164.24(F / E h)$. The same value was obtained with ANSYS $^{\circledR}$ program and the variational method, both presented here.

This is known as a benchmark result where there is a dominant bending behaviour in the thin shell limit. The vertical displacement along the length of the cylinder is shown in Fig.5 where two different finite element approaches (this one with ANSYS ${ }^{\circledR}$ and as published by [7]) and the mixed formulation are compared. Results are similar to the ones obtained by [9].

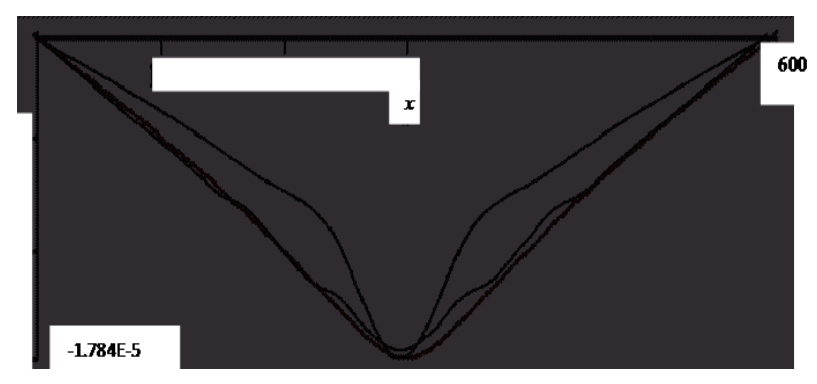

Figure 7. Vertical displacement along the cylinder length (as in [7], presented solutions ANSYS ${ }^{\circledR}$ and mixed formulation)

\section{Conclusions}

The presented numerical tools have shown a good agreement altogether when analysing a recurrent problem in piping engineering, the structural behaviour of thin walled cylindrical pipes subjected to radial loads. The variational solution based on functional analysis, though initially demanding some work with closed form analytic methods, has the advantage of leading to a quite simple computational solution by computer implementation and is fitted to other boundary conditions problems. As the proposed solution deals with analytical formulations higher order derivatives are possible giving continuous and realistic results while in finite elements this is not possible. Moreover, the results match consistently with corresponding work in the literature using finite element techniques and commercial codes. The solution accuracy can be improved by easily expanding the number of trigonometric elements considered in the Fourier expansions included in the presented solution.

\section{Acknowledgements}

The authors wish to thank Fundação para a Ciência e Tecnologia for the financial support leading to this publication.

\section{REFERENCES}


[1] O. C. Zienkiewicz, The Finite Element Method, 3rd Ed McGraw-Hill Co. NY, 1979

[2] E. Oñate, Calculo de Estructuras pro el Metodo de Elementos Finitos CIMNE Barecelona, Spain

[3] F. J. M. Q. Melo, P. M. S. T. Castro, The linear elastic stress analysis of curved pipes under generalized loads using a reduced integration finite ring element, Journal of Strain Analysis, Vol.32, Nº1, 47-59, 1997

[4] A. Millard, R. Roche, Elementary Solutions for the Propagation of Ovalization along Straight Pipes and Elbows. Int J Pressure Vessels Piping, Vol.16, No.1, 101-129, 1984

[5] T. H. Richards, Energy Methods in Strain Analysis, Ellis-Horwood, 1982

[6] C. Wylie, L. Barret. Advanced Engineering Mathematics, 6th ed McGraw-Hill, 1995
[7] E. Fonseca, F. Melo, C. A. Oliveira, Trigonometric function used to formulate a multi-nodal finite tubular element, Mech Res Comm, Vol.34, No.1, 54-62, 2007

[8] R. H. Mac Neal, R.L. Harder, A proposed standard set of problems to test finite element accuracy, Journal of Finite Elements in Analysis and Design, Vol.1, 3-20, 1985

[9] F. Cirak, M. Ortiz, P. Schroder, Subdivision surfaces: a new paradigm for thin shell finite element analysis, Int J Numer Meth Eng- Vol.47, No.12, 2039-2072, 2000

[10] E. Dvorkin, K. J. Bathe, A continuum mechanics based four-node shell element for general non-linear analysis, Eng Comput, Vol.1, 77-88, 1984

[11] R. Kouhia, R. Stenberg, A simple linear nonconforming shell element, IASS-IACM 2000 Fourth International Colloquium on Computational of Shell Spatial Structures, Greece, 2000. 